\title{
Two-body potential model based on cosine series expansion for ionic materials
}

Takuji Oda, ${ }^{1, *}$ William J. Weber, ${ }^{2,3}$ and Hisashi Tanigawa ${ }^{4}$

\author{
${ }^{1}$ Department of Nuclear Engineering, Seoul National University, Republic of Korea \\ ${ }^{2}$ Department of Materials Science \& Engineering, University of Tennessee, USA \\ ${ }^{3}$ Materials Science \& Technology Division, Oak Ridge National Laboratory, USA \\ ${ }^{4}$ Fusion Research and Development Directorate, Japan Atomic Energy Agency, Japan
}

*email: oda@snu.ac.krＴEL:+82-2-880-8988

\begin{abstract}
:
A method to construct a two-body potential model for ionic materials with a Fourier series basis is examined. In this method, the coefficients of cosine basis functions are uniquely determined by solving simultaneous linear equations to minimize the sum of weighted mean square errors in energy, force and stress, where firstprinciples calculation results are used as the reference data. As a validation test of the method, potential models for magnesium oxide are constructed. The mean square errors appropriately converge with respect to the truncation of the cosine series. This result mathematically indicates that the constructed potential model is sufficiently close to the one that is achieved with the non-truncated Fourier series and demonstrates that this potential virtually provides minimum error from the reference data within the two-body representation. The constructed potential models work appropriately in both molecular statics and dynamics simulations, especially if a two-step correction to revise errors expected in the reference data is performed, and the models clearly outperform two existing Buckingham potential models that were tested. The good agreement over a broad range of energies and forces with first-principles calculations should enable the prediction of materials behavior away from equilibrium conditions, such as a system under irradiation.
\end{abstract}




\section{Introduction}

Force-field methods such as classical molecular statics (MS) and molecular dynamics (MD) methods are important computational techniques in condensed matter physics. Since the interatomic interactions are approximately described by simplified equations, the so-called potential model, the computational cost for energy and force evaluations is largely reduced compared with first-principles calculations. Due to this approximation, however, the accuracy of simulation results is affected by the quality of the potential model. Hence, developing a potential model that appropriately describes a phenomenon of interest is a key step to obtain realistic results by a force-field method.

Constructing a potential model is generally composed of four steps, after determining a phenomenon and a system to be studied.

(1) Gather reference data toward which a potential model is optimized.

(2) Choose an interaction model frame: two-body representation, three-body representation, etc.

(3) Define a function formula of the potential model: Lennard-Jones, Morse, Buckingham, etc, in the two-body representation, for example.

(4) Determine a method to optimize the parameters in the model function, and then apply it.

At each step, some errors are incorporated into the potential model. Regarding step (1) for a solid material, early studies referenced experimental data on crystallographic, thermal, mechanical and vibrational properties of the target system. Since the number of model parameters must be sufficiently smaller than the number of reference data, a simple model function was required. This means that steps (2) and (3) were restricted by step (1). This situation was changed by the advancement of first-principles calculation. Nowadays, one can evaluate energies and forces of a system of interest with a high accuracy. Thus, a great number of reference data can be prepared to employ a complex model formula.

In the past few decades, several sophisticated model functions were suggested, such as the Tersoff [1] and Brenner [2] potentials for covalent materials, variable charge models [3,4] for ionic materials, and embedded-atom model (EAM) [5] and its modified version (MEAM) [6] for metallic materials. In addition, more complex potential models composed by multiple model functions, like the REAX-FF model [7] have been becoming popular to cover various chemical forms of substances at once. These sophisticated models were derived considering the physics and the chemistry of interatomic interactions. 
Even for such sophisticated model functions, however, their numerical accuracy is still limited because available function space is restricted by prepared function formulas, namely errors in step (3). Hence, as another approach, mathematics-oriented methodologies using a more flexible representation of potential energy have been attracting increasing attention [8], such as potential energy fitting with interpolated moving least-squares methods (IMLS) [9,10], Gaussian approximation potentials (GAP) [11] based on Gaussian process regression [12], a method based on a linear regression using the least absolute shrinkage and selection operator (LASSO) [13], and generalized neural network representation [14]. These methods can construct a potential model which yields energies and forces close to first-principles calculations, such as a GAP model for tungsten [15]. However, the computational cost is largely increased [16].

For some applications that require long-time and/or large-system simulations, including simulation of irradiation damage processes in nuclear materials, a potential model that realizes reasonable accuracy with a low computational cost is practically vital. Regarding the computational cost, the two-body representation has the best advantage. Although the two-body representation is apparently insufficient for covalent and metallic systems, it is expected to provide reasonable accuracy for ionic materials, as many two-body potential models have been successfully developed. However, most two-body potential models for ionic materials are still composed by simple empirical functions such as the Buckingham and the Morse potential models, which can only use a small part of the function space available for the two-body representation. If the function space is fully utilized, the quality of two-body potential models may be largely improved.

In the present study, therefore, we examine a mathematics-oriented method to construct two-body potential models for ionic materials with a quasi-complete basis functions. For this purpose, a Fourier series is employed as the basis functions, instead of empirical model functions. The coefficients of the basis functions are uniquely and straightforwardly determined by solving linear simultaneous equations to minimize the sum of weighted mean square errors in energy, force and stress, where first-principles calculation results are used as the reference data. Since the Fourier series is reasonably regarded as a complete orthogonal system in the function space that two-body potential models belong to, if the fitting error from the reference data is appropriately converged with respect to the truncation of the Fourier series, this method provides a potential model adequately close to the one that has the minimum model error within the two-body representation. In short, the method constructs a virtually best two-body potential model. We may also expect that this method 
will uncover the limitation of two-body potential models without being interrupted by uncertainties caused by steps (3) and (4).

The remaining part of the paper is organized as follows. In chapter 2, the potential model formula and its parameterization method is explained. In chapter 3, first-principles calculation results that were not utilized in the parameterization step are used as the reference data to test validity of the methodology, and the convergence behaviors of the sum of weighted mean square errors in energy, force and stress are shown with respect to the truncation of both the number of reference data and the Fourier series. Magnesium oxide (MgO), which is one of the simplest metal oxides and has a high ionicity, is selected as the test system. In chapter 4 , the quality of the potential model in MS and MD simulations is examined, including mechanical property, melting point, thermal expansion behavior, radial distribution functions and defect energies. In addition, we propose a two-step correction method for constructed potential models in order to revise errors, which are caused by the first-principles calculation, in the reference data. In chapter 5, comparisons on the model quality with existing Buckingham potential models are briefly provided. Finally, the paper is closed with concluding remarks in chapter 6 .

\section{Methodology}

\subsection{Potential model formula}

We consider a periodic function between $-r_{c u t}$ and $+r_{c u t}$, where $r_{c u t}$ represents the cutoff distance of the short-range two-body interaction and is set to $10 \AA$ in the present study. $10 \AA$ is a typical cutoff distance in two-body potential models of ionic materials. Since the functional shape between $-r_{c u t}$ and 0 does not need to have physical meanings, we arbitrarily define this periodic function as an even function so that its first derivative becomes 0 at $r_{c u t}$. It is reasonable to assume that a realistic two-body model function is smooth in the range of possible interatomic distances in condensed matter, e.g. from around $1 \AA$ to $r_{\text {cut }}$. Thus, we can expect that the non-truncated cosine series can compose a two-body potential model that holds the minimum error from the reference data, namely the best model within the two-body representation. With these considerations, the potential energy $\left(E_{p o t}\right)$ in an ionic system is described as follows:

$$
E_{p o t}=\sum_{i, j}^{E w a l d} \frac{q_{i} q_{j}}{r_{i j}}+\frac{1}{2} \sum_{i=1}^{N_{A}} \sum_{j}^{r_{i j}<r_{\text {cut }}}\left[\sum_{k=0}^{N-1} a_{\text {element_i-element }-j, k} \cos \left(\frac{k \pi r_{i j}}{r_{\text {cut }}}\right)\right]+E_{\text {ref }} \text {, and }
$$


$E_{\text {ref }}=n_{M g} e_{M g}+n_{O} e_{O}$,

where $r_{i j}$ is an interatomic distance between two ions $(i$ and $j), q_{i}$ and $q_{j}$ are their ionic charges, $N_{A}$ the number of atoms in a unit simulation cell, $N$ is the number of cosine functions to be utilized for the cosine series representation, and $a_{\text {element_i-element } j, k}$ is the coefficient of $\mathrm{k}$-th cosine function for combination of the elements of $\mathrm{i}$-th and $\mathrm{j}$-th ions (3 kinds of combinations: $\mathrm{Mg}-\mathrm{Mg}, \mathrm{Mg}-\mathrm{O}$ and $\mathrm{O}-\mathrm{O}$ ). In the right-hand side of Eq. (1), the first term denotes the Coulomb interaction, which is evaluated using the Ewald summation method without truncation. In the present study, we simply set $q_{M g}=+2 e$ and $q_{O}=-2 e$, which are typical values for $\mathrm{MgO}$. The second term is of the short-range interaction term composed by a cosine series, where the first summation is taken over the number of atoms in the unit cell $\left(N_{A}\right)$, the second summation is taken over all atoms that are located within the cutoff distance $\left(r_{c u t}\right)$ from i-th ion, including atoms in neighboring image cells, and the third summation is taken over the number of cosine basis functions $(N)$. The multiplication by $1 / 2$ is required to cancel the double counting of atomic combinations. The third term in Eq. (1) is the reference energy, which is needed to appropriately compare energies calculated by a potential model and energies determined by DFT calculation, because absolute energies given by pseudopotential DFT calculation have no clear physical meaning. $E_{r e f}$ is written as the sum of reference energies of atoms involved in a unit simulation cell, where $n_{M g}$ and $n_{O}$ are, respectively, the numbers of $\mathrm{Mg}$ and $\mathrm{O}$ atoms involved in the unit simulation cell, and $e_{M g}$ and $e_{O}$ are, respectively, the reference energies of the $\mathrm{Mg}$ and $\mathrm{O}$ atoms. $e_{M g}$ and $e_{O}$ are independent of atomic configurations.

The model error to be minimized is defined as the sum of the weighted mean square errors in energy, force and stress, referenced to the values determined with first-principles calculation:

$$
\begin{aligned}
& \text { Model_error }=P\left(a_{M g-M g, 0}, \ldots ., a_{M g-M g, \mathrm{~N}-1}, a_{M g-\mathrm{O}, 0}, \ldots . ., a_{M g-\mathrm{O}, \mathrm{N}-1}, a_{\mathrm{O}-\mathrm{O}, 0}, \ldots ., a_{\mathrm{O}-\mathrm{O}, \mathrm{N}-1}, e_{M g}, e_{O}\right) \\
& =\frac{1}{N_{C}} \sum_{i=1}^{N_{C}}\left\{w_{e}\left(E_{p o t}^{i}-E_{Q M}^{i}\right)^{2}+\frac{w_{f}}{3 N_{A}} \sum_{j=1}^{3 N_{A}}\left(F_{p o t}^{i, j}-F_{Q M}^{i, j}\right)^{2}+\frac{w_{s}}{6} \sum_{j=1}^{6}\left(S_{p o t}^{i, j}-S_{Q M}^{i, j}\right)^{2}\right\}
\end{aligned}
$$

where $E, F$ and $S$ denote the energy, the force and the stress, respectively. $N_{C}$ is the number of configurations. The superscript for $E$ is the configuration index $i$, those for $F$ are the configuration index $i$ and the force component index $j$ (3 dimensions for each atom), and those for $S$ are the configuration index $i$ and the stress component index $j(\mathrm{xx}, \mathrm{yy}, \mathrm{zz}, \mathrm{xy}, \mathrm{yz}, \mathrm{zx})$, respectively. $w_{e}, w_{f}$ and $w_{s}$ are the weighting factors for energy, 
force and stress, and their units are $\mathrm{eV}^{-1},(\mathrm{eV} / \AA)^{-1}$, and $\mathrm{eV}^{-1}$, respectively. Note the pressure unit for stress (e.g. $\mathrm{Pa}$ ) was converted to the energy unit $(\mathrm{eV})$ by multiplying by the volume of the simulation cell. In the present study, we set $w_{e}=1, w_{f}=10$ and $w_{s}=0.1$ so that the resultant error contributions from energy, force and stress become roughly comparable in Eq. 3. The subscript "pot" denotes a value determined with the potential model, while " $Q M$ " a value with first-principles calculation. $F_{p o t}$ is calculated from the first derivative of $E_{p o t}$. $S_{p o t}$ is calculated using the conventional version of the virial equation [17].

Then, $(3 N+2)$ simultaneous linear equations are constructed as

$\frac{\partial P}{\partial a_{M g-M g, k}}=0, \frac{\partial P}{\partial a_{M g-O, k}}=0, \frac{\partial P}{\partial a_{O-O, k}}=0, \frac{\partial P}{\partial e_{M g}}=0, \frac{\partial P}{\partial e_{O}}=0$,

where the $k$ index is for $N$ integers (integers from 0 to $N-1$ ). By solving the simultaneous linear equations, we can uniquely achieve a set of $a_{i-j, k}, e_{M g}$ and $e_{O}$ that gives a minimum error from the reference data with the prepared cosine basis functions up to $N$-th cosine series. The influences of pre-determined parameters, such as the ionic charges $\left(q_{M g}\right.$ and $\left.q_{O}\right)$ and the cut-off radius $\left(r_{c u t}\right)$, are not discussed in the present study.

In Eq. 1, if $N$ is set to infinity, the solution of Eq. 4 gives a potential model that is obtained with a non-truncated Fourier cosine series expansion, namely the best two-body potential model that provides the minimum model error given by Eq. 3. In practice, if the model error appropriately converges with respect to the truncation of Fourier cosine series, we can consider that the constructed potential model is virtually the best one. The convergence test result will be examined in chapter 3 .

\subsection{Reference data}

\subsubsection{Basic dataset}

To build up the reference data to be used in the potential model construction, we performed firstprinciples molecular dynamics (FPMD) simulations of a NVT ensemble with $3 \times 3 \times 3 \mathrm{MgO}$ supercells containing 128 atoms. 150000 trajectories were obtained in total, where 50 conditions of different sets of temperature and cell volume were prepared and 3000 trajectories were accumulated for each condition. Regarding the temperature, it ranged from $300 \mathrm{~K}$ to $6000 \mathrm{~K}$, including both solid and liquid phases. The time step was $1 \mathrm{fs}$. The minimum distance that appeared in the FPMD simulations was $2.16 \AA$ for $\mathrm{Mg}-\mathrm{Mg}, 1.54 \AA$ 
for $\mathrm{Mg}-\mathrm{O}$ and $2.12 \AA$ for $\mathrm{O}-\mathrm{O}$ at $3000 \mathrm{~K}$ (around the melting point), and was $1.75 \AA, 1.38 \AA$ and $1.11 \AA$ at $6000 \mathrm{~K}$ (the highest temperature of the present FPMD simulations), respectively, for example. Note that the quality of the potential model is not guaranteed at distances than do not appear in the reference data.

For each trajectory, the total electronic energy (a single data value), forces (384 data as 3 components of 128 atoms), and stresses (6 components) were determined for use as the reference data. All calculations were performed using the VASP code (version 5.3.3) [18] based on density functional theory (DFT), with the projector augmented-wave (PAW) method [19] and plane-wave basis set. The Perdew-Burke-Ernzerhof (PBE) exchange-correlation functional [20] was utilized. The outer eight and six electrons, respectively for magnesium ( $2 p$ and $3 s$ ) and for oxygen ( $2 s$ and $2 p)$, were explicitly handled, while the others were regarded as core electrons handled with PAW potentials. All calculations were performed under the spin-unpolarized condition. The plane-wave cutoff energy was set at $500 \mathrm{eV}$, and k-point sampling of band energies was done with $2 \times 2 \times 2$ Monkhorst-Pack grid [21]. The error due to the plane-wave energy cutoff and the k-point sampling grid size was estimated to be less than around $1 \mathrm{meV} /$ atom for the energy difference and $0.01 \mathrm{eV} / \mathrm{A}$ for the force.

In addition to this basic dataset, we prepared two extra reference datasets as follows, in order to improve the potential model quality on mechanical properties and on defect energies.

\subsubsection{Additional dataset 1: for mechanical properties}

Elastic constant are related to energy (or stress) responses against small volume changes around the equilibrium volume. Since the magnitude of such energy changes is trivial, they may not be sufficiently reproduced by the present model construction method. In addition, the computational setting for the FPMD calculations is insufficient for evaluation of elastic constants, because the quality of the plane waves are changed by the volume change, which is related to Pulay stress [22], and the degree of the wrap around error in the fast Fourier transformation (FFT) of wave functions may be altered by volume change.

Therefore, we additionally performed first-principles calculation to reinforce the reference dataset for mechanical properties. For this purpose, we consider isotropic volume changes and uni-axial volume changes. The former is related to the bulk modulus and the latter to Young's modulus and Poisson's ratio. An FFT 
setting to avoid the wrap around errors and a well-converged setting of the plane-wave cutoff energy $(800 \mathrm{eV})$ were utilized with a large k-point sampling grid $(3 \times 3 \times 3)$. The first-principles calculation results were then processed so that they become compatible with the basic dataset regarding the equilibrium volume at $0 \mathrm{~K}$ and the total energy at the equilibrium volume.

\subsubsection{Additional dataset 2: for defect energies}

As discussed below, the formation energies of $\mathrm{O}$ Frenkel pairs are not well reproduced by the potential model established with the basic dataset and the additional dataset-1. Therefore, we obtained firstprinciples calculation data for Frenkel pairs. Several systems containing an O Frenkel pair or a Mg Frenkel pair were prepared with various vacancy-interstitial separation distances. A geometry optimization was first performed. Then, with the optimized defect structures, we performed FPMD calculations at $300 \mathrm{~K}$ and $1200 \mathrm{~K}$. By this procedure, the information on the optimized defect structures and some thermally-distorted defect structures are involved in the additional dataset- 2 .

The calculations were performed under the spin-polarized condition, although spin polarization did not occur in the optimized structures and in the following FPMD calculation. Except for this spin setting, the computational setting identical to the basic dataset was utilized. The system charge was set neutral.

\section{Validation results}

\subsection{Convergence to the number of reference data}

Prior to a convergence test of the model error for the truncation of cosine series, we performed another convergence test on the number of FPMD trajectories utilized as the reference data. The results are shown in Fig. 1, where the model error is expressed by the root mean square (RMS) errors from the FPMD data in energy, force and stress. For this evaluation, we divided the FPMD data into two groups: (i) 75000 trajectories, all or some of which are used as the reference data in the potential model construction, and (ii) 75000 trajectories, which are not included in the model construction stage but are utilized to evaluate RMS errors in Fig. 1. The RMS errors are normalized with the one that was determined for the potential model constructed with all 75000 trajectories in dataset (i). The number of cosine basis functions was fixed at 50 for all cases here. Good convergence of RMS errors with respect to the number of reference FPMD data is 
evident in Fig. 1. The errors induced by the number of reference data is reduced to less than $10 \%$ with around 10000 FPMD trajectories and to few \% with around 20000 FPMD trajectories.

Hereafter, the number of referenced FPMD trajectories is fixed at 75000 for all cases.

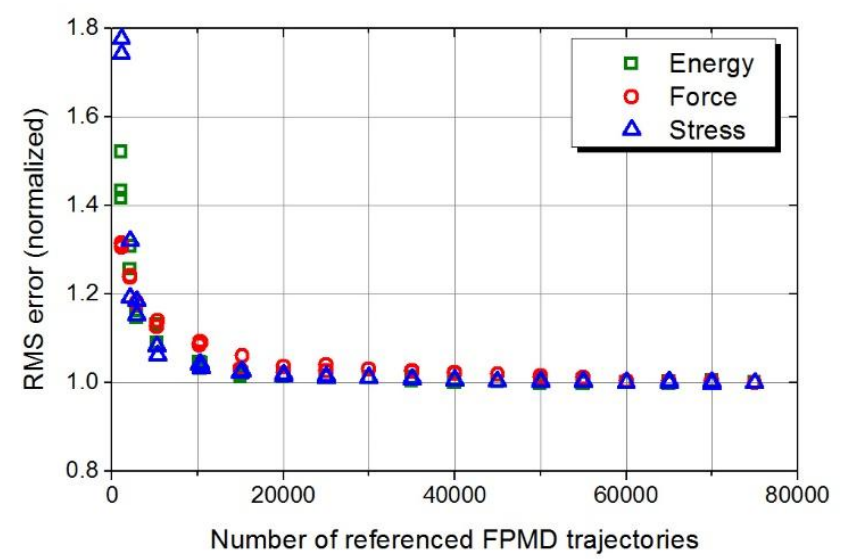

Fig. 1. Convergence of model errors regarding the number of reference data. RMS error values are normalized with the ones of 75000 reference trajectories.

\subsection{Convergence to the number of cosine basis functions}

In order to check the error convergence with respect to the truncation of cosine series, the model error was determined as a function of the number of cosine basis functions, as shown in Fig. 2. A good convergence is seen with 25 cosine basis functions, indicating that the truncated cosine series up to more than the 25 -th cosine basis function can compose a potential model that is sufficiently close to the one that is achieved with a non-truncated Fourier cosine series. It is therefore reasonable to consider that the converged RMS errors represent errors intrinsic to the "two-body interaction model with the formal-charge explicit Coulomb interaction" for $\mathrm{MgO}$, which is $6.7 \mathrm{meV} /$ atom in energy, $0.29 \mathrm{eV} / \AA$ in force and $3.1 \mathrm{eV}$ in stress as the RMS error. Note that these intrinsic errors depend on several conditions, such as the contents of the reference data, the weighting factors, the ionic charges and the cut-off radius of the two-body interaction.

In Fig. 2, the stress has a larger error than the energy and the force. There are two reasons for this. First, a wrong relation in elastic constants $\left(C_{12}=C_{44}\right)$ is forcibly maintained in the two-body potential models, namely the Cauchy relation, which should cause significant errors. Second, the Pulay stress [22] is not negligible in the current DFT calculation setting: the error due to the Pulay stress in $\mathrm{xx}$, yy and zz components 
of the stress tensor was estimated as $-2.7 \times 10^{8} \mathrm{~Pa}$. We made a Pulay stress correction [22] by simply subtracting these values as is sometimes done. However, this simple correction seemed insufficient for the structures that appeared at high temperatures, where the cell volumes were significantly enlarged from that optimized at the static condition. These errors may be technically eliminated or diminished, although we do not pursue it in the present study, by including a many-body interaction term in the potential model for the former, and utilizing a larger plane-wave basis sets in first-principles calculation for the latter.

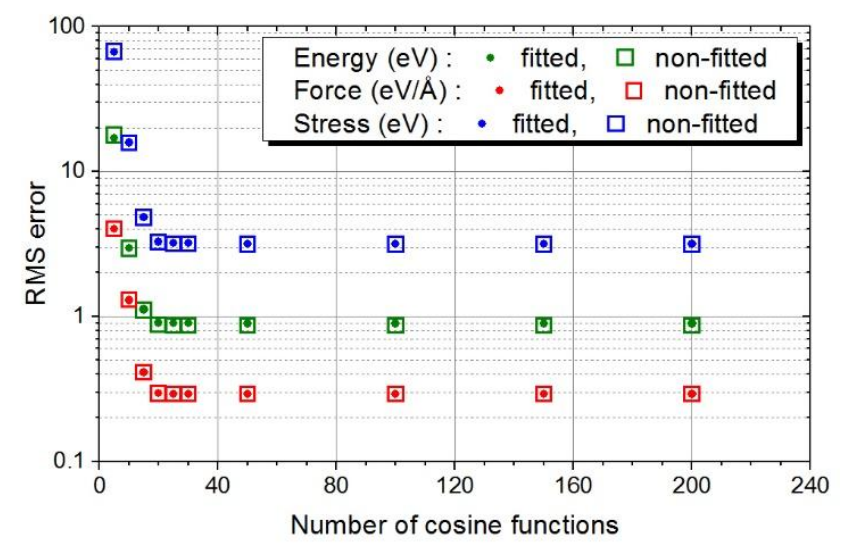

Fig. 2. Convergence of model errors regarding the number of cosine basis functions. Marks of 'fitted' indicate the errors from the FPMD data that were used in the potential model construction, while marks of 'non-fitted' indicate the errors from the FPMD data that were not used.

\subsection{Comparison with the reference data}

In Fig. 3, comparisons of energy, force and stress between values determined with the first-principles calculation and those obtained with the constructed potential model $(N=50)$ are given. Good agreement is widely seen, ranging over $200 \mathrm{eV}$ (corresponding to $1.5 \mathrm{eV} /$ atom) in energy, from $-8 \mathrm{eV} / \AA$ to $+8 \mathrm{eV} / \AA$ in force, and from - $200 \mathrm{eV}$ to $100 \mathrm{eV}$ (corresponding to from -10 GPa to $20 \mathrm{GPa}$, approximately) in stress.

In the energy comparison, there is a tendency that better agreement is achieved for lower energies, which means that the solid phase is better described than the liquid phase. This result indicates that manybody interactions, which are neglected in the present model, have more significant contributions in the liquid phases than in solid phases. 

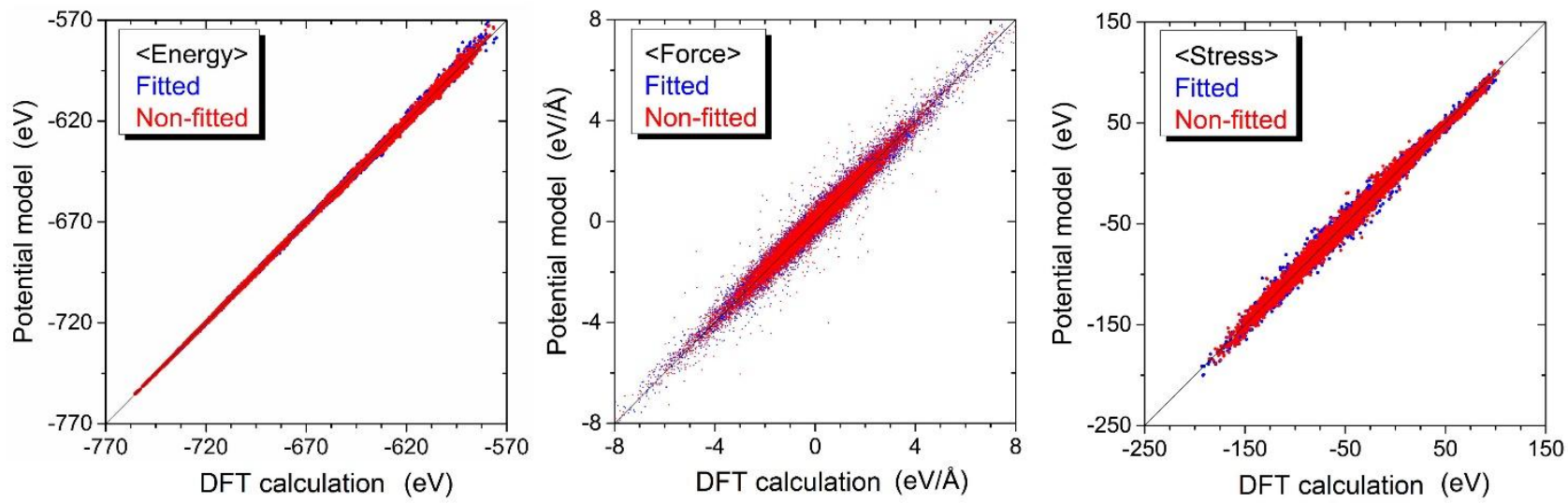

Fig. 3. Comparisons between the potential model and the DFT calculation in energy, force and stress.

\subsection{Shape of the constructed model}

The shapes of the constructed potential functions $(N=50)$ are drawn in Fig. 4. Good convergences of the potential energy to 0 at $r=r_{\text {cut }}(10 \AA)$ are observed for all three combinations, although no dumping function is utilized. Specifically, the potential energy at $r=10 \AA$ is $-0.000028 \mathrm{eV}$ for $\mathrm{Mg}-\mathrm{Mg}$ interaction, $0.00012 \mathrm{eV}$ for $\mathrm{Mg}-\mathrm{O}$ interaction, and $-0.00070 \mathrm{eV}$ for $\mathrm{O}-\mathrm{O}$ interaction.

The wavelength of the highest-frequency cosine function is around $0.41 \AA$ with $N=50$. Since numerous fitting targets are involved and emerged interatomic distances densely cover interatomic distances of around $2 \AA$ and above, even the use of 200 cosine functions, in which the minimum wavelength is around $0.10 \AA$, does not cause a significant wavy structure in shape. The smooth shapes are evident in Fig. 5 for both energy and force, where the absolute values of energy and force are also given on a logarithm scale to emphasize fine structures. Strictly speaking, there is a fine structure at around $9.8 \AA$ in $\mathrm{Mg}-\mathrm{Mg}$ interaction. However, because the absolute values of energy and force variations in the fine structure are small (on the range of $10^{-4}-10^{-6} \mathrm{eV}$ in energy and $10^{-3}-10^{-4} \mathrm{eV} / \AA$ in force), it should hardly affect MS and MD simulations in practice.

The shapes of the obtained functions are qualitatively different from those of existing two-body potential models, e.g. in comparison with reference [23], if the Coulomb interaction terms are excluded. Specifically, the constructed functions behave as to weaken the Coulomb interaction expressed by the fullcharge model: the anion-anion and cation-cation interactions are mostly attractive, while the anion-cation interaction is repulsive. Nevertheless, involving the Coulomb interaction term makes the model functions 
reasonable in overall appearance: the anion-anion and cation-cation are repulsive over the whole range, and the anion-cation is attractive up to around a certain distance $(\sim 1.6 \AA)$ and then turns to strongly repulsive at shorter distances.

Those two results, namely the smooth shape and the reasonable attractive/repulsive interactions, indicate that physical characteristics of the interatomic interactions are appropriately reproduced in the constructed model functions. We also confirmed that the shape of the potential model with $N=50$ is almost identical to those with $N=25$ and with $N=200$, which again suggests a nice convergence with respect to the truncation of cosine series.

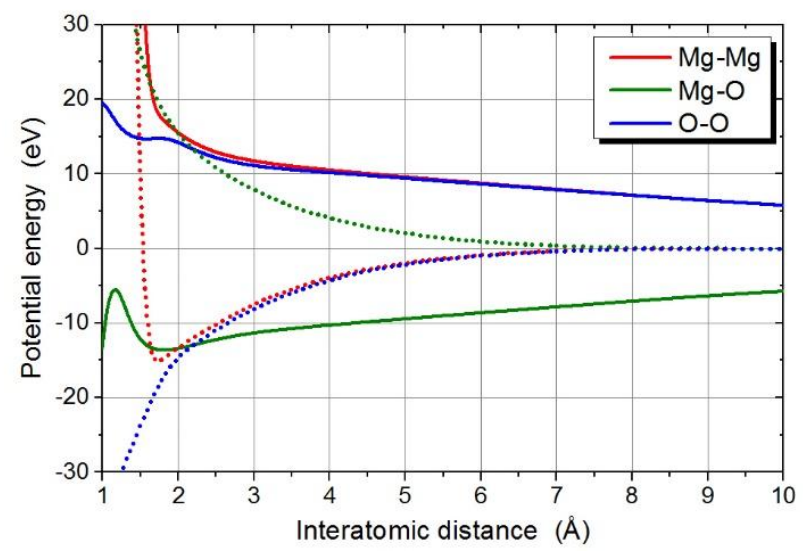

Fig. 4. Functional shapes of potential energy in the constructed potential model. The ones presented by solid lines include the Coulomb interaction term, while the ones by dotted lines exclude it.

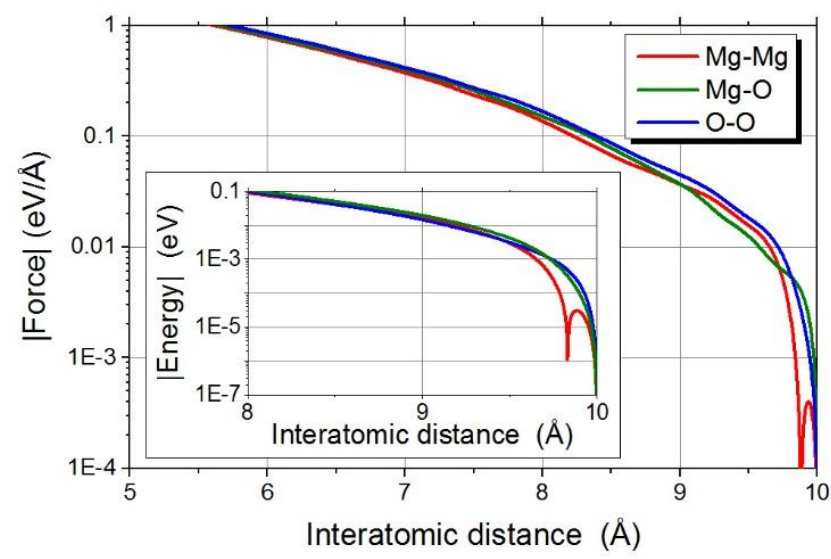

Fig. 5. Detailed shapes of energy and force functions of the constructed potential model. 


\section{Performance in MS and MD simulations}

According to the result of chapter 3 , we constructed three potential models using 50 cosine basis functions: PMl by referring only the basic dataset, which is the same condition as set in the chapter 3; PM2 by referring the basic dataset and the additional dataset-1; PM3 by referring the basic dataset, the additional datset- 1 and the additional dataset- 2 . In this chapter, results of performance tests on elastic constants, melting point, thermal expansion behavior, radiation distribution functions and defect energies are presented.

\subsection{Elastic constants}

The results of performance tests for elastic constants are given in Table 1. All calculations were performed using the GULP code [24]. Apparently, the quality of PM1 is unsatisfactory. To improve the quality, the additional dataset-1 was also referred to in the construction of PM2 and PM3, as explained in the section 2.2.2. Consequently, better agreement with the DFT calculation values is achieved within the limitation of the two-body representation, namely, $C_{12}=C_{44}$.

It is known that the DFT calculation with the PBE functional generally underestimates the elastic constants and overestimates the lattice constants [25]. These errors of the DFT calculation are clearly propagated to the constructed potential models. To revise the errors, we propose a two-step correction method. In the first step, the interatomic distance in the potential model functions is linearly scaled so that the lattice constant agrees with the experimental value. In the second step, the potential energy is linearly scaled so that the bulk modulus agrees with the experimental value. This two-step correction alters the potential model formula of Eq. 1 as follows

$$
\begin{aligned}
& E_{p o t}^{c o r}(\mathbf{r})=s_{p}\left\{\sum_{i-j}^{3}\left\{\frac{q_{i} q_{j}}{\left(r_{i-j} / s_{r}\right)}+\sum_{k=0}^{N-1} a_{i-j, k} \cos \left(\frac{k \pi\left(r_{i-j} / s_{r}\right)}{r_{\text {cut }}}\right)\right\}+E_{r e f}\right\} \\
& =\sum_{i-j}^{3}\left\{\frac{\left(\sqrt{s_{p} / s_{r}} q_{i}\right)\left(\sqrt{s_{p} / s_{r}} q_{j}\right)}{r_{i-j}}+\sum_{k=0}^{N-1} s_{p} a_{i-j, k} \cos \left(\frac{k \pi r_{i-j}}{s_{r} r_{c u t}}\right)\right\}+s_{p} E_{r e f} \\
& s_{r}=\frac{l_{\text {exp }}}{l_{\text {pot } 0}}, s_{p}=\frac{B_{\text {exp }}}{B_{p o t ~}},
\end{aligned}
$$


where $s_{r}$ and $s_{p}$ are respectively the scaling factors of the first and the second correction steps. The scaling factors are obtained by Eq. 6, where $l_{\text {exp }}$ and $l_{\text {poto }}$ are the lattice constant in the experiment and in the simulation using the constructed potential model, respectively, and $B_{e x p}$ and $B_{p o t l}$ are the bulk moduli in the experiment and in the simulation using the potential model after the first correction, respectively. Consequently, the cutoff distance is scaled by $s_{r}$ and the ionic charges are scaled by $\sqrt{s_{p} / s_{r}}$. We utilized $l_{\text {exp }}$ $=4.189 \AA$ and $B_{\text {exp }}=169.8 \mathrm{GPa}$, which are not raw experimental values $(4.207 \AA$ and $162.5 \mathrm{GPa})$ but the modified values to exclude the zero-point phonon effects [26,27]. Hereafter, the new potential models revised with the two-step correction are called PM2C and PM3C, which were respectively obtained by correcting PM2 and PM3. The two new models give better agreement with the experimental values on the lattice constant and the elastic constants, as shown in Table 1.

Table 1. Comparison in some fundamental material properties of $\mathrm{MgO}$. The experimental data are taken from references [28-30]. The lattice constant and bulk modulus excluding the zero-point phonon effects (ZP) are taken from reference [27]. The elastic constants excluding the ZP are approximately calculated by multiplying the experimental values with 1.045 , which is the scaling factor of the bulk modulus.

\begin{tabular}{|c|c|c|c|c|c|c|c|c|c|}
\hline & \multicolumn{2}{|c|}{ Experiment } & \multirow[t]{2}{*}{ DFT } & \multicolumn{5}{|c|}{ Potential model } \\
\hline & & raw data & excl. ZP & & PM1 & PM2 & PM3 & PM2C & PM3C \\
\hline \multicolumn{2}{|c|}{ Lattice constant $(\AA)$} & 4.207 & 4.189 & 4.261 & 4.266 & 4.261 & 4.261 & 4.189 & 4.189 \\
\hline \multirow{3}{*}{$\begin{array}{c}\text { Elastic } \\
\text { constants } \\
(\mathrm{GPa})\end{array}$} & $C_{11}$ & 297.0 & 310.3 & 270.3 & 199.1 & 267.5 & 269.1 & 309.7 & 308.5 \\
\hline & $C_{12}$ & 95.2 & 99.5 & 88.5 & 109.4 & 86.2 & 87.6 & 99.8 & 100.4 \\
\hline & $C_{44}$ & 155.7 & 162.7 & 140.6 & 109.4 & 86.2 & 87.6 & 99.8 & 100.4 \\
\hline \multicolumn{2}{|c|}{ Bulk modulus (GPa) } & 162.5 & 169.8 & 149.1 & 139.3 & 146.7 & 148.1 & 169.8 & 169.8 \\
\hline \multicolumn{2}{|c|}{ Melting point (K) } & 3098 & - & - & $\sim 2700$ & 2700 & $\sim 2900$ & $\sim 3100$ & $\sim 3100$ \\
\hline
\end{tabular}




\subsection{Melting point}

The melting point at $1 \times 10^{5} \mathrm{~Pa}$ was determined by MD using the LAMMPS code [31]. The stability of mixed solid-liquid phases was checked for 200 ps. In Table 1, “ 2700 K” means that the mixed phase becomes a sole solid phase at $2600 \mathrm{~K}$ while it becomes a sole liquid phase at $2800 \mathrm{~K}$ after the MD simulation of $200 \mathrm{ps}$. As the melting point is located between $2600 \mathrm{~K}$ and $2800 \mathrm{~K}$, in this case, the intermediate value $(\sim 2700 \mathrm{~K})$ is provided in Table 1.

The simple two-step correction also improved the reproductivity of the melting point. The melting point is slightly underestimated in the potential models without the two-step correction. As expected from Eq. 5, the two-step correction strengthens the interatomic interaction, which increases the stability of solid phases more than liquid phases regarding the potential energies. As a result, the melting point is increased, and thus the agreement with experiment is improved. The underestimation of the melting point in the non-corrected models (PM1, PM2 and PM3) seems to come from the characteristic error of the PBE functional that interatomic interactions are underestimated.

\subsection{Thermal expansion}

Thermal expansion behaviors at $1 \times 10^{5} \mathrm{~Pa}$ were examined by MD using the LAMMPS code. The result of PM3, PM2C and PM3C are given in Fig. 6. The results of PM1 and PM2 are similar to that of PM3. Due to the overestimation of the lattice constant at $0 \mathrm{~K}$, which reflects the error of the PBE functional, PM3 does not reproduce the experimental date.

On the other hand, PM2C and PM3C nicely reproduce the thermal expansion except at low temperatures, at which the zero-point phonon effect is significant. The disagreement at low temperatures is reasonable because the zero-point phonon effect is not taken into account in classical MD. The good performance of $\mathrm{PM} 2 \mathrm{C}$ and $\mathrm{PM} 3 \mathrm{C}$ in the melting point and the thermal expansion behavior demonstrates that the two-step correction, which is designed to correct the errors caused by the PBE functional in the reference data, nicely works despite its simplicity. 


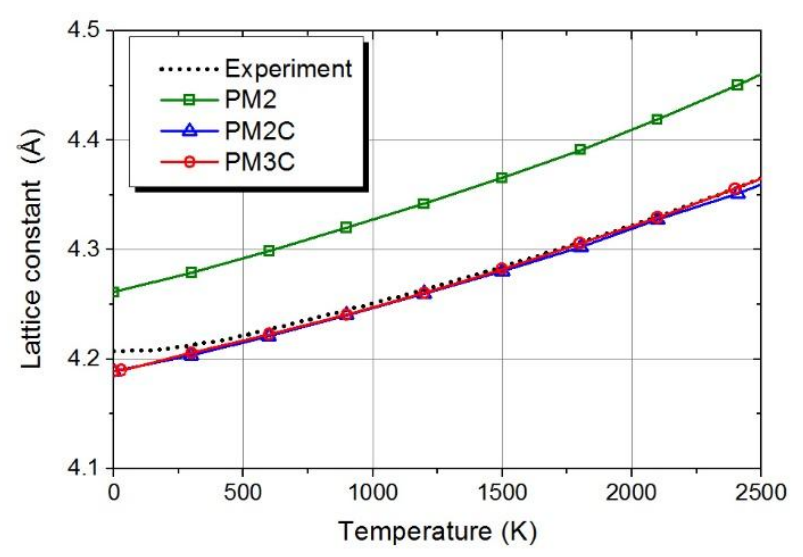

Fig. 6. Comparison in the thermal expansion behavior of $\mathrm{MgO}$. The experimental data are derived from references [28,29].

\subsection{Radial distribution functions}

Radial distribution functions (RDFs) of O-O at $1800 \mathrm{~K}$ and $3600 \mathrm{~K}$ are given in Fig. 7. The former is of a solid phase and the latter of a liquid phase. The $3 \times 3 \times 3$ supercells (from the primitive cell, containing 128 atoms) that were used in the FPMD calculations for the reference data generation were employed to acquire the RDFs for both FPMD and classical MD under the NVT ensemble. In the FPMD calculations, the average pressure in the system was around $-0.3 \mathrm{GPa}$ at $1800 \mathrm{~K}$ and around $10 \mathrm{GPa}$ in $3600 \mathrm{~K}$, respectively. Comparable pressures were also induced in the MD calculations with PM2. The RDFs obtained in classical MD with PM2 agree well with those obtained in FPMD (labelled as "DFT"). We also confirmed good agreement in the RDFs of $\mathrm{Mg}-\mathrm{Mg}$ and $\mathrm{Mg}-\mathrm{O}$, including the ones at $300 \mathrm{~K}$. In addition, RDFs in $6 \times 6 \times 6$ supercells (from the conventional cell, containing 1728 atoms) were evaluated with the potential model in order to check the effect of the small $3 \times 3 \times 3$ supercells in the RDF evaluation. No significant cell-size effect was found up to RDFs of $6 \AA$ radius. 


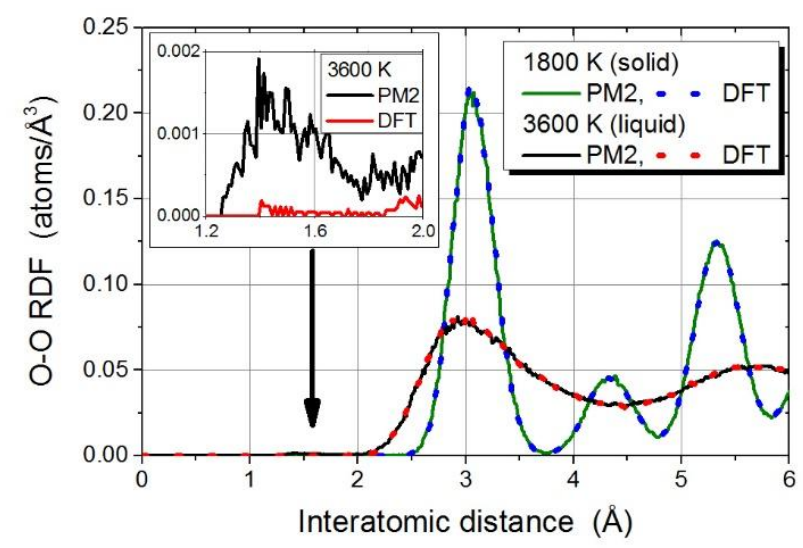

Fig. 7. Comparison in radial distribution functions of O-O at $1800 \mathrm{~K}$ and $3600 \mathrm{~K}$.

In the O-O RDF of FPMD calculation for the liquid phase, some non-zero values are seen in short interatomic distances $(\sim 1.4 \AA)$. This distribution suggests the formation of the peroxide ion $\left(\mathrm{O}_{2}{ }^{-}\right)[32,33]$ according to the interatomic distance. The formation of the peroxide ion is reproduced by PM1 and PM2, although the distribution was larger than DFT. On the other hand, the peroxide ion is not formed with PM3. The reason for this difference will be discussed in the next section. Peroxide ions were also formed with PM2C at $3600 \mathrm{~K}$ while not in PM3C, as expected from the results of PM2 and PM3.

\subsection{Defect energies}

Three types of point defects were examined: Mg Frenkel pair, O Frenkel pair and Schottky defect. Several defect configurations differing in the distance between the vacancy and the interstitial atom for Frenkel pairs and in the distance between $\mathrm{Mg}$ vacancy and $\mathrm{O}$ vacancy for Schottky defects were considered. The calculations were done using the GULP code and the VASP code.

The formation energies determined with the potential models and those determined by the DFT calculations are compared in Fig. 8. PM1 shows similar results to PM2. The formation energies of Mg Frenkel pairs and Schottky defects are well described even with PM2, although the first-principles calculation data of defect structures was not referred to in its construction. This result indicates that the chemical environments around Mg Frenkel pairs and Schottky defects are not largely different from the chemical environment of atoms in perfect crystals and liquids, which were used as fitting target of PM2. 
In contrast, the formation energies of O Frenkel pairs are largely underestimated by PM2, while PM3 provides good results. A previous DFT calculation [33] showed that an interstitial atom forms peroxide-type split interstitials and the isolated interstitial is less stable in the group II monoxides, including $\mathrm{MgO}$. The peroxide-type interstitials are also obtained in the present calculation of O Frenkel pairs.

Accordingly, there are two types of peroxides in MgO: one appears in the solid phase as a defect and the other appears in the liquid phase. It seems that the chemical environment of these two peroxides are different, and the function space of the two-body representation is not large enough to describe both by one model. Indeed, in the construction of PM3, we first could not correct the underestimation of the O Frenkel pair energy, even by using the additional dataset- 2 . To correct the underestimation, we needed to decrease the weight of liquid information in the model fitting process of PM3. However, by doing so, peroxides disappeared in liquids. We could not construct a model that can nicely describe the peroxides in both solid and in liquid phases.

There is a clear difference between PM2 and PM3 in the potential energy curve of O-O interaction at around $1.5 \AA$, as shown in Fig. 9. This distance range corresponds to the O-O distance in the peroxides. PM3 gives a more repulsive interaction between two $\mathrm{O}$ atoms at this distance range than PM2, which results in a larger formation energy for O Frenkel defect and a lower stability of peroxide in liquid phases.

Regarding the influence of the two-step correction, the correction just linearly scales the defect formation energies. For example, the defect formation energies calculated with PM2C and PM3C are 1.0999 and 1.0891 times as large as those calculated with PM2 and PM3, respectively. The scaling factors (1.0999 for PM2C and 1.0891 for PM3C) are equal to $s_{p} / s_{r}{ }^{2}$ in Eq. 5. PM3C gives better agreement with the DFT calculation than PM3, although this result should be just accidental. PM2C also provides slightly better agreement with the DFT calculation than PM2, again probably by accident. 


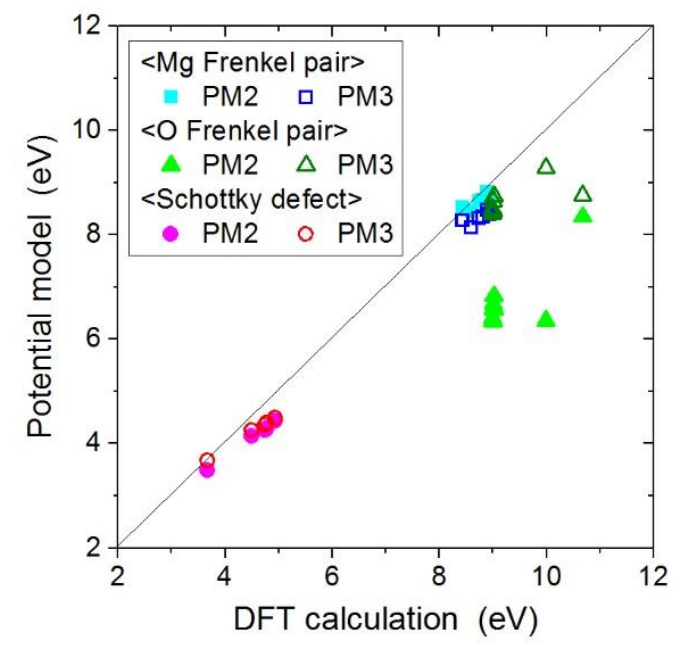

Fig. 8. Comparison in defect formation energies.

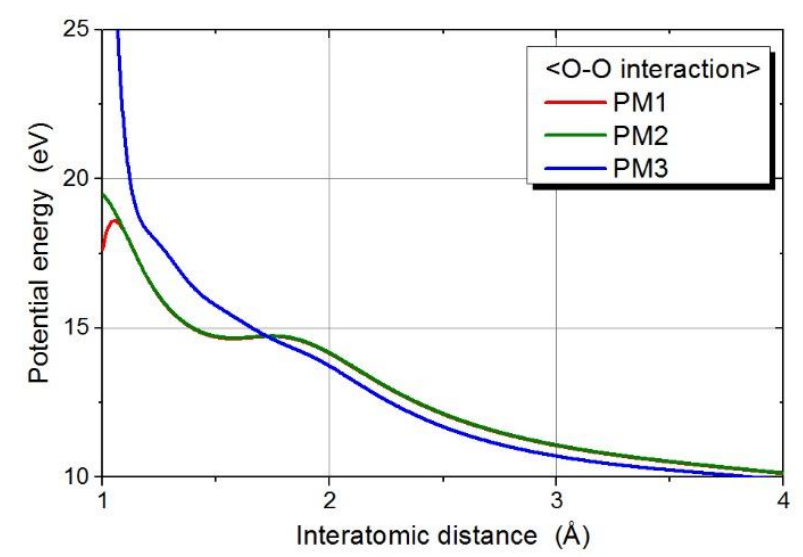

Fig. 9. Comparison in O-O potential energy curves of PM1, PM2 and PM3. The Coulomb interaction terms are included.

\section{Comparison with classical models}

In this chapter, we briefly compare the performance of the present potential models and that of fullcharge Buckingham models. For this purpose, two models are selected: one produced by Lewis and Catlow (L-C) [34] and the other by Ball and Grimes (B-G) [35]. In order to make the comparisons within the twobody representation, the core-shell model of the L-C model was converted to the corresponding rigid-ion model by omitting the core-shell interaction. Note that the omission of the core-shell interaction degrades the 
performance in elastic constants evaluation, e.g. the Cauchy relation $\left(C_{12}=C_{44}\right)$ is obtained; but this omission is often performed to reduce the computational cost in MD simulations [35].

The first comparison is on energy and force where the DFT results are used as the reference data. Fig. 10 shows the comparison result on randomly selected 1470 structures for energy and 70560 components for force. For the energy comparison, because not absolute values but relative values are physically meaningful, energy values in the L-C and B-G models are uniformly shifted so that the RMS errors from DFT data are minimized. The evaluated RMS errors are $211 \mathrm{meV} /$ atom for energy and $1.9 \mathrm{eV} / \AA$ for force in L-C model and $289 \mathrm{meV} /$ atom and $2.1 \mathrm{eV} / \mathrm{A}$ in B-G model, respectively, which are much larger than those of the cosineseries models (6.7 meV/atom and $0.29 \mathrm{eV} / \mathrm{A}$ for PM1). As a general trend observed in Fig. 10, interatomic interactions are overestimated in the L-C and B-G models. The overestimation can be assigned to the Coulomb interaction of the full-charge model. As shown in Fig. 4, the cosine-series model largely reduces the Coulomb interaction over a wide distance range, while the $\mathrm{L}-\mathrm{C}$ and $\mathrm{B}-\mathrm{G}$ models do not so.

In spite of the low accuracy in the energy and force description, Buckingham models generally show good performance in MS and MD simulations. The lattice constant, elastic constants and the melting point determined by the L-C and B-G models are listed in Table 3, and the thermal expansion behavior is given in Fig. 11. The quality of L-C model is not largely inferior to the cosine-series models in these properties. However, considering the results given in Fig. 10, the good reproducibility in these properties is not a result of accurate energy and force description but rather from appropriate cancellation of errors in the energy and force description. Although DFT calculations in themselves are not free of errors, a fair agreement with the DFT energy, force and stress should be important when a force field method is utilized to predict materials behavior away from equilibrium, or perfect crystal, conditions, such as in a system under irradiation, extreme stress, shock, high pressure, etc. From this viewpoint, the cosine-series models apparently outperform the two Buckingham models.

Another clear superiority of the cosine-series models is found in the description of defects. Table 3 compares the formation energy of O Frenkel defect of different configurations. If we just look at the defect formation energy, the L-C Buckingham model works well. However, the structure of the most stable defect and relative stabilities of different defect configurations are largely different from the results of DFT calculations. In contrast, PM3 gives fair agreement with the DFT calculation results. 
Strictly speaking, however, the relative stabilities between $\langle 111\rangle$ and $\langle 110\rangle$ peroxides are wrongly evaluated even with PM3. Indeed, we could not construct a potential model to make <111> peroxide more stable than $\langle 110\rangle$ peroxide. This would be another limitation of the two-body representation.

Finally, the performance test results are summarized in Table 3. As a whole, PM2C and PM3C outperform the others, considering the excellent agreement in the mechanical properties, the melting point and the thermal expansion with experiment, as well as good description of the peroxide in a solid phase by PM3C and in a liquid phase by PM2C. Since the errors caused by the DFT calculation were corrected on purpose in PM2C and PM3C, the increases in the energy fitting error is not problematic. Note that the force fitting error is still quite comparable with the DFT calculation, because force is related to energy difference, not the absolute value of energy.

In future applications, we recommend PM3C for simulation of solid phases, including the study of irradiation damage, and PM2C for simulation of liquid phases including solid-liquid phase transition. PM2C should also nicely work in solid simulation, if the concentration of Frenkel pairs is low. Indeed, since the formation energies are large, Frenkel pairs are hardly formed in $\mathrm{MgO}$ at thermodynamic equilibrium states of reasonable temperature and pressure conditions.

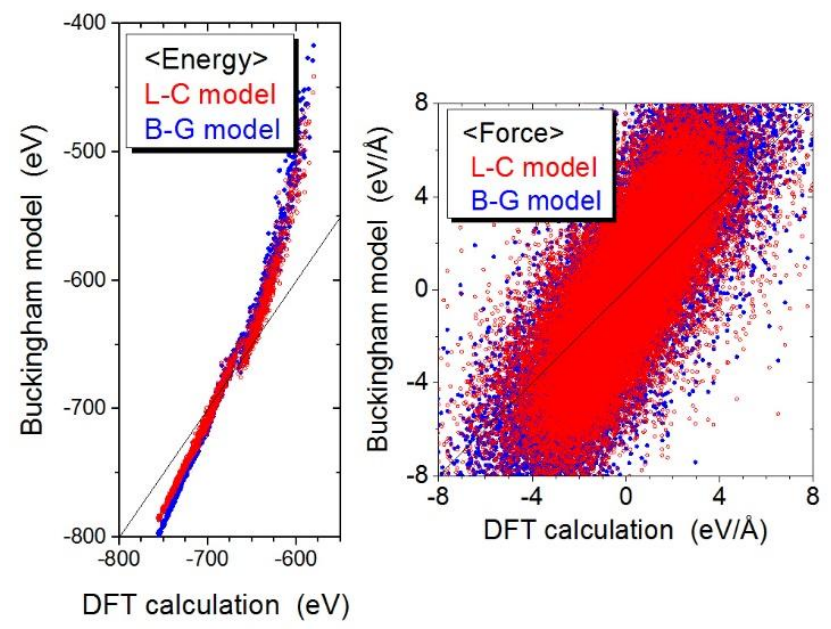

Fig. 10. Comparisons between the two Buckingham models and the DFT calculation in energy (left) and force (right). 
Table 2. Fundamental material properties of $\mathrm{MgO}$ determined by the Buckingham models. The melting point of B-G model was just confirmed to be larger than $3600 \mathrm{~K}$.

\begin{tabular}{|c|c|c|c|}
\hline \multicolumn{2}{|c|}{} & L-C & B-G \\
\hline \multicolumn{2}{|c|}{ Lattice constant $(\AA)$} & 4.211 & 4.209 \\
\hline \multirow{2}{*}{$\begin{array}{c}\text { Elastic constants } \\
(\mathrm{GPa})\end{array}$} & $\mathrm{C}_{11}$ & 296.0 & 374.3 \\
\cline { 2 - 4 } & $\mathrm{C}_{12}$ & 166.5 & 172.1 \\
\cline { 2 - 4 } & $\mathrm{C}_{44}$ & 166.5 & 172.1 \\
\hline \multicolumn{2}{|c|}{ Bulk modulus $(\mathrm{GPa})$} & 209.6 & 239.5 \\
\hline \multicolumn{2}{|c|}{ Melting point (K) } & $\sim 3100$ & $>3600$ \\
\hline
\end{tabular}

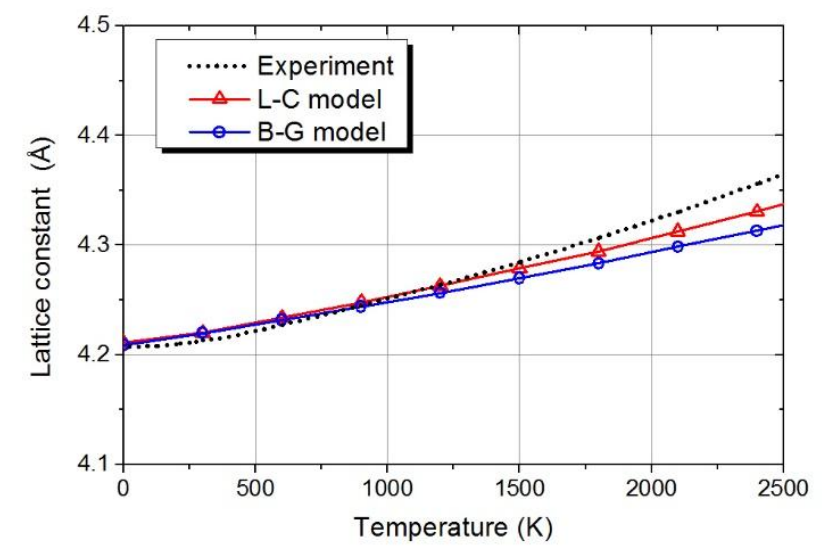

Fig. 11. Thermal expansion behavior evaluated with the two Buckingham models.

Table 3. Comparison in the formation energies of O Frenkel pairs of different interstitial configurations. The bold fonts indicates the most stable configuration. The values given with parentheses were obtained with fixing the peroxide coordinates to the DFT result, in order to avoid changing to the most stable configuration.

\begin{tabular}{|l|l|l|l|l|}
\hline & DFT & PM3 & L-C & B-G \\
\hline$<100>$ peroxide & 10.8 & 9.16 & $(15.3)$ & $(32.3)$ \\
\hline$<110>$ peroxide & 9.22 & $\mathbf{8 . 9 6}$ & $(14.3)$ & $(27.5)$ \\
\hline$<111>$ peroxide & $\mathbf{8 . 9 8}$ & $(9.17)$ & $(14.9)$ & $(28.9)$ \\
\hline Interstitial & 10.7 & 9.40 & $\mathbf{9 . 9 4}$ & $\mathbf{1 2 . 8}$ \\
\hline
\end{tabular}


Table 4. Summary of the performance test. The percentages show the difference from the DFT calculation result. The percentages on the defect formation energies show the mean square error from the DFT calculation result over tested defects given in Fig. 8. 'over' and 'under' respectively indicate overestimation and underestimation. ' $O$ ' and ' $x$ ' respectively indicate the appearance and non-appearance of peroxides in a liquid phase.

\begin{tabular}{|l|l|l|l|l|l|l|l|}
\hline & \multicolumn{5}{|c|}{ Cosine-series } & \multicolumn{2}{c|}{ Buckingham } \\
\hline & PM1 & PM2 & PM2C & PM3 & PM3C & L-C & B-G \\
\hline Energy error (meV/atom) & 6.7 & 6.7 & 29 & 7.5 & 10 & 211 & 289 \\
\hline Force error (eV/A) & 0.29 & 0.29 & 0.33 & 0.32 & 0.34 & 1.9 & 2.1 \\
\hline Lattice constant at 0 K (\%) & 1.8 & 1.7 & 0 & 1.7 & 0 & 0.53 & 0.48 \\
\hline Elastic constant, C 11 (\%) & -36 & -14 & -0.19 & -13 & -0.6 & -4.6 & 21 \\
\hline Melting point (\%) & -13 & -13 & \pm 3 & -6 & \pm 3 & \pm 3 & $>16$ \\
\hline Thermal expansion & over & over & good & over & good & under & under \\
\hline Peroxides in liquid & 0 & 0 & 0 & $\times$ & $\times$ & $\times$ & $\times$ \\
\hline Mg Frenkel pair (\%) & 2.5 & 1.3 & 9.0 & 4.7 & 3.8 & 20 & 40 \\
\hline O Frenkel pair (\%) & 27 & 27 & 20 & 7.1 & 4.7 & 11 & 37 \\
\hline Schottky defect (\%) & 10 & 9.2 & 1.7 & 6.8 & 1.8 & 22 & 49 \\
\hline
\end{tabular}

\section{Conclusion}

We demonstrate that a truncated cosine series can construct a two-body potential model that produces energies, forces and stresses reasonably comparable with DFT calculation over a wide temperature range for $\mathrm{MgO}$, which was chosen as a typical example of ionic materials. The shape of the constructed potential model is smooth and looks physically reasonable. Adequate convergences of model errors were confirmed with respect to: (i) the number of the reference data utilized in the model construction process and (ii) the number of the cosine basis functions. The latter convergence indicates that the constructed model is sufficiently close to the best two-body potential model that has the minimum error from the reference data within the two-body 
representation. Thus, the resultant error can be regarded as the error intrinsic to the two-body representation, although the error depends on several conditions, such as the contents of the reference data, the weighting factors, the ionic charges, and the cut-off radius of the two-body interaction, which need to be addressed in future works.

We also confirmed good performance of the constructed potential model in molecular statics and dynamics simulations, specifically on the lattice constants, the melting point, the thermal expansion behavior, the radial distribution functions, and the defect energies. At the same time, however, some limitations of the two-body representation are observed, such as less accurate energies in the liquid phases, the relatively large errors in stress evaluation, and the less accurate simultaneous description of peroxides in the solid phase and in the liquid phase.

In force field methods, there is always a tradeoff between the accuracy and the computational cost (speed). Two-body interaction models have a great advantage in computational cost. Note that even though many cosine functions are utilized in the present model, numerical tabulation of energy/force values affords a minimal computational cost, as with other two-body models. In addition, for ionic models, the accuracy of the two-body representation is reasonably good, and the present method can maximize the accuracy of two-body potential model, as demonstrated in this study. Thus, if low computational cost is needed and if an ionic material is investigated, the present method is a promising and straightforward way to construct a quality twobody potential model.

The cosine-series two-body model is also easily coupled with other potential models. For example, it can be employed with a variable charge model. In this case, since a virtually best two-body model is obtained with given variable-charge parameters, the parameter space that we need to search out is largely reduced. For an application where a higher accuracy is needed, we may combine two types of potential models, similar to methods of combined quantum mechanical and molecular mechanical potential (QM/MM) [36,37]. For example, a combination of the GAP model [11] and cosine-series two-body model, which employs a GAP model for a region where a higher accuracy in energy is needed and utilizes a cosine-series two-body model for a region where a lower accuracy is acceptable, may manage both the accuracy and the speed for ionic materials. 


\section{Acknowledgements}

This research was supported by BK 21 plus project, the Center for Advanced Research in Fusion Reactor Engineering (CARFRE) and 2014 Aspiring Researcher Program of Seoul National University (SNU). One of the authors (WJW) was supported by the U.S. Department of Energy, Office of Science, Basic Energy Sciences, Materials Sciences and Engineering Division. The calculations in this research were carried out using the HELIOS supercomputer system at the Computational Simulation Center of the International Fusion Research Center (IFERC-CSC) in Japan. 


\section{References}

[1] J. Tersoff, Phys. Rev. B 37 (1988) 6991.

[2] D. Brenner, Phys. Rev. B 42 (1990) 9458.

[3] K.A. Van Genechten, W.J. Mortier, P. Geerlings, J. Chem. Phys. 86 (1987) 5063.

[4] A.K. Rappe, W. a. Goddard, J. Phys. Chem. 95 (1991) 3358.

[5] M. Daw, M. Baskes, Phys. Rev. B 29 (1984) 6443.

[6] M. Baskes, J. Nelson, A. Wright, Phys. Rev. B 40 (1989) 6085.

[7] A.C.T. van Duin, S. Dasgupta, F. Lorant, W.A. Goddard, J. Phys. Chem. A 105 (2001) 9396.

[8] C.M. Handley, J. Behler, Eur. Phys. J. B 87 (2014) 152.

[9] D.H. McLain, Comput. J. 17 (1974) 318.

[10] G.G. Maisuradze, D.L. Thompson, A.F. Wagner, M. Minkoff, J. Chem. Phys. 119 (2003) 10002.

[11] A.P. Bartók, M.C. Payne, R. Kondor, G. Csányi, Phys. Rev. Lett. 104 (2010) 136403.

[12] C.E. Rasmussen, C.K.I. Williams, Gaussian Processes for Machine Learning, MIT Press, 2006.

[13] A. Seko, A. Takahashi, I. Tanaka, Phys. Rev. B 90 (2014) 024101.

[14] J. Behler, M. Parrinello, Phys. Rev. Lett. 98 (2007) 146401.

[15] W.J. Szlachta, A.P. Bartók, G. Csányi, Phys. Rev. B 90 (2014) 104108.

[16] S.J. Plimpton, A.P. Thompson, MRS Bull. 37 (2012) 513.

[17] M.J. Louwerse, E.J. Baerends, Chem. Phys. Lett. 421 (2006) 138.

[18] G. Kresse, Phys. Rev. B 54 (1996) 11169.

[19] P.E. Blöchl, Phys. Rev. B 50 (1994) 17953.

[20] J.P. Perdew, K. Burke, M. Ernzerhof, Phys. Rev. Lett. 77 (1996) 3865.

[21] H.J. Monkhorst, J.D. Pack, Phys. Rev. B 13 (1976) 5188.

[22] G.P. Francis, M.C. Payne, J. Phys. Condens. Matter 2 (1990) 4395.

[23] T.S. Bush, J.D. Gale, C.R.A. Catlow, P.D. Battle, J. Mater. Chem. 4 (1994) 831.

[24] J.D. Gale, J. Chem. Soc. Faraday Trans. 93 (1997) 629. 
[25] G. Csonka, J. Perdew, A. Ruzsinszky, P. Philipsen, S. Lebègue, J. Paier, O. Vydrov, J. Ángyán, Phys. Rev. B 79 (2009) 155107.

[26] P. Hao, Y. Fang, J. Sun, G.I. Csonka, P.H.T. Philipsen, J.P. Perdew, Phys. Rev. B 85 (2012) 014111.

[27] J. Sun, M. Marsman, G.I. Csonka, A. Ruzsinszky, P. Hao, Y.-S. Kim, G. Kresse, J.P. Perdew, Phys. Rev. B 84 (2011) 035117.

[28] L.S. Dubrovinsky, S.K. Saxena, Phys. Chem. Miner. 24 (1997) 547.

[29] G.K. White, J. Appl. Phys. 37 (1966) 430.

[30] C.S. Zha, H. Mao, R.J. Hemley, Proc. Natl. Acad. Sci. U. S. A. 97 (2000) 13494.

[31] S. Plimpton, J. Comput. Phys. 117 (1995) 1.

[32] S.C. Abrahams, J. Kalnajs, Acta Crystallogr. 7 (1954) 838.

[33] S.C. Middleburgh, K.P.D. Lagerlof, R.W. Grimes, J. Am. Ceram. Soc. 96 (2013) 308.

[34] G. V Lewis, C.R. a Catlow, J. Phys. C Solid State Phys. 18 (1985) 1149.

[35] G. Henkelman, B. Uberuaga, D. Harris, J. Harding, N. Allan, Phys. Rev. B 72 (2005) 115437.

[36] A. Warshel, M. Levitt, J. Mol. Biol. 103 (1976) 227.

[37] M.J. Field, P.A. Bash, M. Karplus, J. Comput. Chem. 11 (1990) 700. 


\section{Figure and table captions}

Fig. 1. Convergence of model errors regarding the number of reference data. RMS error values are normalized with the ones of 75000 reference trajectories.

Fig. 2. Convergence of model errors regarding the number of cosine basis functions. Marks of 'fitted' indicate the errors from the FPMD data that were used in the potential model construction, while marks of 'non-fitted' indicate the errors from the FPMD data that were not used.

Fig. 3. Comparisons between the potential model and the DFT calculation in energy, force and stress.

Fig. 4. Functional shapes of potential energy in the constructed potential model. The ones presented by solid lines include the Coulomb interaction term, while the ones by dotted lines exclude it.

Fig. 5. Detailed shapes of energy and force functions of the constructed potential model.

Fig. 6. Comparison in the thermal expansion behavior of $\mathrm{MgO}$. The experimental data are derived from references $[28,29]$.

Fig. 7. Comparison in radial distribution functions of O-O at $1800 \mathrm{~K}$ and $3600 \mathrm{~K}$.

Fig. 8. Comparison in defect formation energies.

Fig. 9. Comparison in O-O potential energy curves of PM1, PM2 and PM3. The Coulomb interaction terms are included.

Fig. 10. Comparisons between the two Buckingham models and the DFT calculation in energy (left) and force (right).

Fig. 11. Thermal expansion behavior evaluated with the two Buckingham models.

Table 1. Comparison in some fundamental material properties of $\mathrm{MgO}$. The experimental data are taken from references [28-30]. The lattice constant and bulk modulus excluding the zero-point phonon effects (ZP) are taken from reference [27]. The elastic constants excluding the ZP are approximately calculated by multiplying the experimental values with 1.045 , which is the scaling factor of the bulk modulus.

Table 2. Fundamental material properties of $\mathrm{MgO}$ determined by the Buckingham models. The melting point of B-G model was just confirmed to be larger than $3600 \mathrm{~K}$.

Table 3. Comparison in the formation energies of O Frenkel pairs of different interstitial configurations. The bold fonts indicates the most stable configuration. The values given with parentheses were obtained with 
fixing the peroxide coordinates to the DFT result, in order to avoid converging to the most stable configuration.

Table 4. Summary of the performance test. The percentages show the difference from the DFT calculation result. The percentages on the defect formation energies show the mean square error from the DFT calculation result over tested defects given in Fig. 8. 'over' and 'under' respectively indicate overestimation and underestimation. ' $O$ ' and ' $x$ ' respectively indicate the appearance and non-appearance of peroxides. 
$<$ Potential function> Two-body model by a Fourier cosine series 2-body potential by Fourier series for ionic materials

Determine the cosine coefficients by solving linear equations to minimize errors from DFT results
$<$ Reference data>

DFT results on energy/force/stress
Good error convergence to the truncation of cosine series

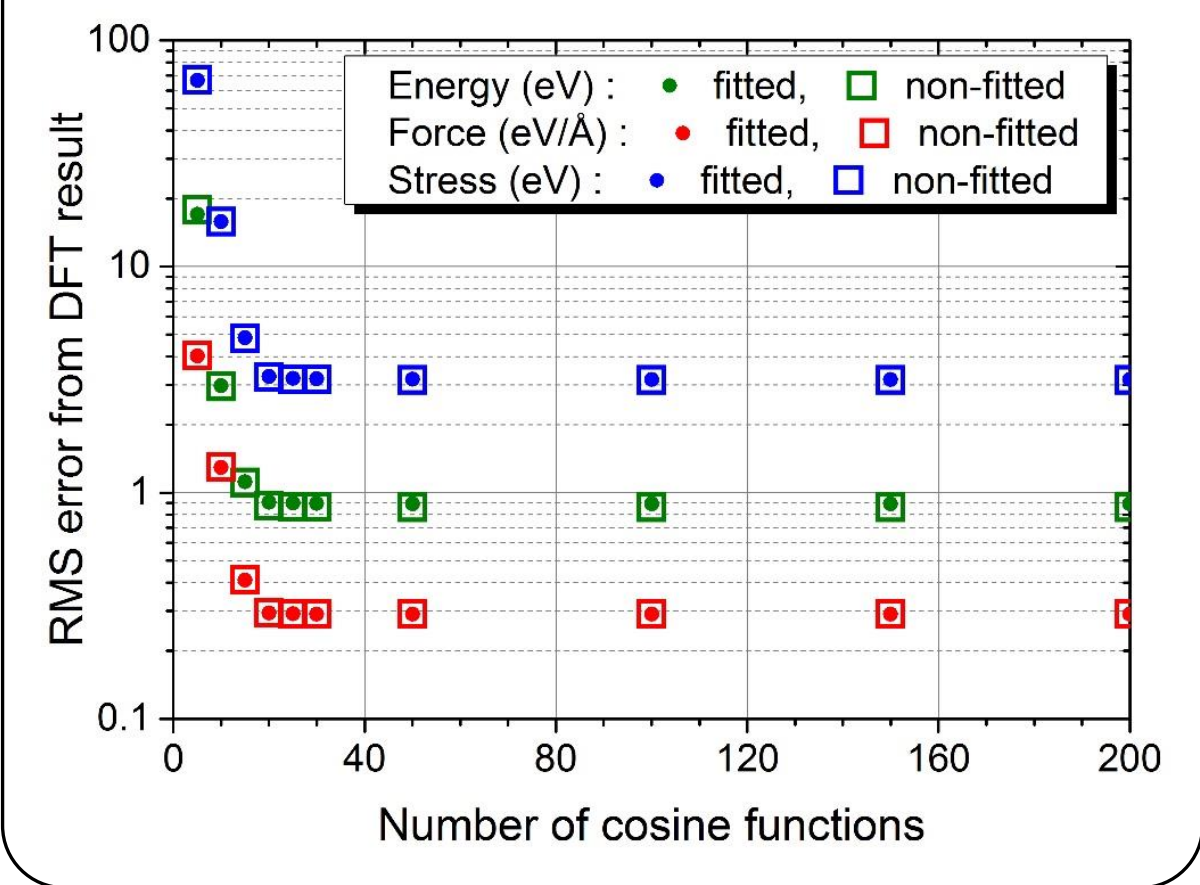

[Advantages]

$\checkmark$ A virtually "best" two-body potential model.

$\checkmark$ Reasonably accurate for ionic materials.

(tested with MgO)

$\checkmark$ Better perform than classical models, e.g. Buckingham.
Good performance in MS and MD

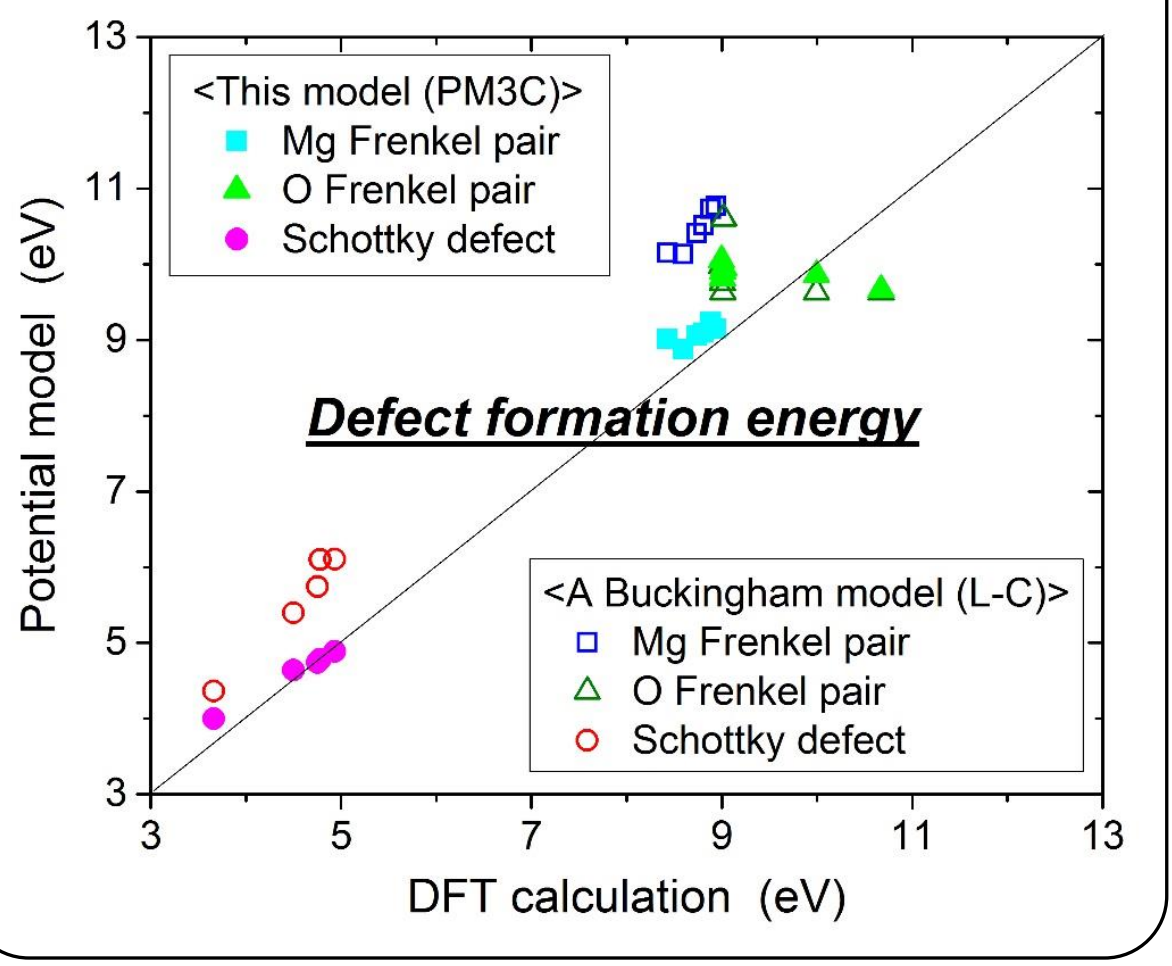

